\title{
Equivalent circuit for RF flexural free-free MEMS resonators
}

\author{
M. Mastrangeli · A. Nannini · D. Paci · F. Pieri
}

(C) Springer Science + Business Media, LLC 2006

\begin{abstract}
A method to extract a lumped-parameter equivalent circuit for a free-free flexural MEMS resonator, based on the Euler-Bernoulli beam equation and exploiting a modal analysis approach, is presented. The dynamic behaviour predicted by the equivalent circuit is compared with FEM simulations, and the effect of a geometrical mismatch is investigated as well. The resonance frequency and the quality factor are correctly predicted. The method could be used for more complex systems of interconnected beams. The circuit can be used as a quick and intuitive analysis tool for the systemlevel designer and to allow the simulation of the device in a system-level design environment.
\end{abstract}

Keywords Equivalent circuit $\cdot$ Lumped-parameters · MEMS flexural resonators - FEM simulations .

Euler-Bernoulli beam

\section{Introduction}

Compact models for MEMS components, which operate on multiple physical domains, are required for efficient simulation of complex systems, including MEMS devices as well as analog and digital circuits. Consequently, a large amount of MEMS literature has been devoted to the development of such models (often based on a lumped-parameter approach) and to their integration in the flow of system simulation and design [1-3]. In this respect, electrical lumped-parameter

M. Mastrangeli · A. Nannini · D. Paci $(\bowtie)$ · F. Pieri

Dipartimento di Ingegneria dell'Informazione, Università di Pisa,

Via Caruso 16, 56122 Pisa Italy

e-mail: dario.paci@iet.unipi.it

D. Paci

CNR-IEIIT Sezione di Pisa, via Caruso 16, 56122 Pisa Italy equivalent circuits are especially useful. They reduce the number of state variables of the model and they can be implemented in a language for behavioural modelling [3], but also on more established and conventional (from the point of view of the electronic design) circuit simulators. This last aspect is relevant not only because circuit simulators are readily available in a design environment, but also because the equivalent circuit gives to the designer a quick insight into the component behaviour regardless of the underlying physical working principle, leading to a tighter interaction between the system-level and the device designer.

Among MEMS devices, electromechanical resonators have been proposed as fundamental components for RF filters and mixers [4], as well as frequency references for RF oscillators [4-6]. Several different designs, based on torsional, flexural, and bulk vibrational modes, have been presented, and equivalent circuits for a number of different designs have been proposed $[5,7,8]$, although a general approach to equivalent circuit extraction is not established yet.

In this paper we present a method to extract a lumpedparameter equivalent circuit for laterally actuated free-free flexural resonators. FEM simulations of the frequency response of devices with different dimensions were performed to validate the model and investigate the effect of design fabrication error.

\section{Resonator structure and operation}

The free-free flexural resonator is shown in Fig. 1: the mechanical structure is composed by a main beam with free ends, suspended at the nodal points of its first resonance mode by two support clamped-clamped beams. The resonator is driven into motion by an electrostatic force generated by the 
Fig. 1 Schematic structure of the free-free resonator. The vertical main beam and horizontal support beams are represented. Darker squares are anchors to the substrate

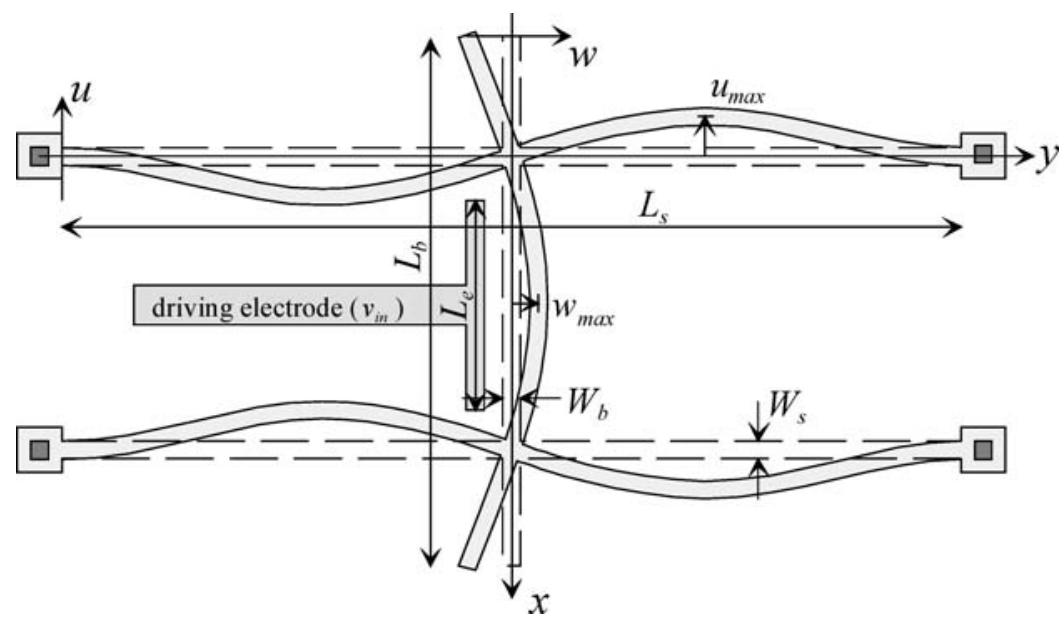

voltage $V_{\text {in }}$; an output electrode, properly biased with a DC voltage $V_{\text {bias }}$, is used to collect the current, due to the change in the output capacitance, produced by resonator motion.

The actual device was fabricated in the thick-film technology THELMA, developed by STMicroelectronics. A SEM photograph of the device is shown in Fig. 2.

Support beams are connected to the main beam at their middle point and are designed to resonate on their second resonance mode at the same frequency of the main beam (matching condition), so that their deformed shape has a node (i.e. fixed point) at the intersection. As a consequence, at least ideally, they do not exert any bending moment on the main beam. This allows higher quality factors with respect to more conventional structures $[9,10]$. To predict the effect of design and fabrication errors on the quality factor and on the resonance frequency, it is interesting to investigate the dynamic behaviour of the structure when the matching condition is not respected, i.e. when the supports are longer (or shorter) than the optimum length. The equivalent circuit presented in the following was developed to model this aspect as well.

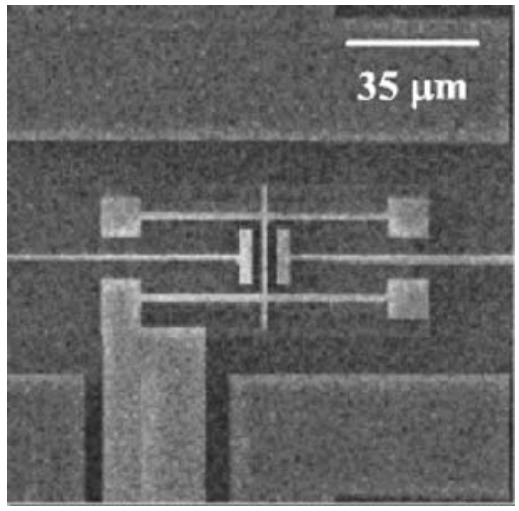

Fig. 2 SEM image of the resonator

\section{Equivalent circuit}

The resonator in Fig. 1 can be decomposed into three beams; each of them can be modelled according to the EulerBernoulli beam equation, as the beams can be considered slender (i.e. with their length more than five times their width). The more complete Timoshenko beam theory [11], used for thick beams, is computationally tougher and does not lead to a compact equivalent circuit. Results from EulerBernoulli model have shown a reasonably good agreement with measurements on similar devices [12].

The deflections $w(x, t)$ (for the main beam) and $u(y, t)$ (for the supports) can be written as a linear superposition of the equation solutions found by modal analysis [7], each of them corresponding to a different resonance frequency. By calling $\Phi_{n}(x)$ and $\Psi_{n}(y)$ these solutions, i.e. the mode-shapes of the $n^{\text {th }}$ resonance mode of the main beam and of the supports, respectively, the displacements are thus expressed as

$$
\begin{aligned}
& w(x, t)=\sum_{n=1}^{+\infty} \xi_{n}(t) \Phi_{n}(x) \\
& u(y, t)=\sum_{n=1}^{+\infty} \eta_{n}(t) \Psi_{n}(y)
\end{aligned}
$$

where $\xi_{n}(t)$ and $\eta_{n}(t)$ are generalized displacements. A closed analytical form for each mode-shape can be calculated. Substitution of Eqs. (1) in the Euler-Bernoulli equation leads, for each resonance mode, to the following ordinary differential equation:

$M_{n} \ddot{\xi}_{n}(t)+D_{n} \dot{\xi}_{n}(t)+K_{n} \xi_{n}(t)=P_{n}$

where $P_{n}$ is a generalized force, and $M_{n}, K_{n}$ and $D_{n}$, are the equivalent lumped mass, stiffness and damping respectively. These parameters can be derived by integrating along the beam length the relevant distributed parameter (mass density, 
flexural stiffness, and damping coefficient) weighted with the $n^{\text {th }}$ mode-shape. $P_{n}$ is related to the electrostatic force, and thus it is proportional to the driving voltage $V_{\text {in }}$ through a coupling factor $\Gamma_{n}$.

Equation (2) is formally equivalent to the one describing an RLC series circuit, once the mappings $D_{n} \rightarrow R_{n}, M_{n} \rightarrow$ $L_{n}, 1 / K_{n} \rightarrow C_{n}$ are performed. The generalized force (generalized velocity $\dot{\xi}_{n}$ ) is then interpreted as a voltage (current). The input voltage $V_{\text {in }}$ is coupled with the equivalent circuit of each mode by an ideal transformer of turn ratio $\Gamma_{n}$.

If the quality factor of the resonator is high enough, and the input voltage is narrowband around the first resonance frequency of the main beam, we can suppose that the first generalized displacement $\xi_{1}(t)$ is much larger than those of the higher modes. Consequently, only the first RLC series of the infinitely many implied by Eq. (2) will be included in the equivalent circuit. The same cannot be said of the supports because their length can be different from the optimum (matching) length. While this hypothesis greatly simplifies the equivalent circuit, it is not strictly necessary to the development of the following passages.

To model the dynamic properties of the full system, the behaviour of the nodal points (which are defined by the condition $\Phi_{1}(x)=0$ ) has to be taken into account. We assume a rigid connection, i.e., we suppose that the angle between the main beam and the supports remains square during deflection. This hypothesis can be written simply as:

$$
\left.\frac{\partial w(x, t)}{\partial x}\right|_{x=\hat{x}_{i}}=\left.\frac{\partial u(y, t)}{\partial y}\right|_{y=\hat{y}_{i}}
$$

where $\left(\hat{x}_{i}, \hat{y}_{i}\right)$ are the coordinates of the nodal point. Substitution of Eqs. (1) into Eq. (3) gives

$$
\sum_{n=1}^{\infty} \Phi_{n}^{\prime}(\hat{x}) \xi_{n}(t)=\sum_{n=1}^{\infty} \Psi_{n}^{\prime}(\hat{y}) \eta_{n}(t)
$$

Solving for the first generalized displacement of the main beam we get:

$$
\xi_{1}(t)=\frac{\sum_{n=1}^{\infty} \Psi_{n}^{\prime}(\hat{y}) \eta_{n}(t)}{\Phi_{1}^{\prime}(\hat{x})}-\frac{\sum_{n=2}^{\infty} \Phi_{n}^{\prime}(\hat{x}) \xi_{n}(t)}{\Phi_{1}^{\prime}(\hat{x})} .
$$

Because of the above discussion, the second term in Eq. (5) can be neglected. If we define a coupling coefficient $\Pi_{n}$ between the main beam and the $n^{\text {th }}$ mode of the support beam as

$$
\Pi_{n}=\frac{\Psi_{n}^{\prime}(\hat{y})}{\Phi_{1}^{\prime}(\hat{x})}
$$

Equation (5) becomes:

$\xi_{1}(t)=\sum_{n=1}^{\infty} \Pi_{n} \eta_{n}(t)$

Equation (7) holds also for the first time derivative of the generalized displacements. This leads to a straightforward electrical representation: the equivalent RLC circuit of the $n^{\text {th }}$ mode of the support beams is coupled to the first mode of the main beam through an ideal transformer of turn ratio $\Pi_{n}$ (Fig. 3). We note here that this approach could be generalized to more complex systems of interconnected beams, although the resultant equivalent circuit would eventually become so complex as to be impractical for many scopes. It can be shown that the expression in Eq. (6) can be written also as

$\Pi_{n}=\frac{\lambda_{c c, n}}{\lambda_{f f, 1}} \frac{L_{b}}{L_{s}} F\left(\lambda_{c c, n}, \lambda_{f f, 1}\right)$

where $L_{b}, L_{s}$ are the lengths of the main beam and the support beam, $\lambda_{c c, n}, \lambda_{f f, 1}$ are the $n^{\text {th }}$ eigenvalue of the support beam and the first eigenvalue of the main beam, respectively; the function $F\left(\lambda_{c c, n}, \lambda_{f f, 1}\right)$ can be easily derived from the analytical expressions of the mode-shapes [11]. It can be shown that $F$ is bounded for $\lambda_{c c, n} \rightarrow \infty(n \rightarrow \infty)$.

The existence of a nodal point at the centre of the support beams is compatible with their even resonance modes only; thus in Eq. (7) only even $n$ are considered. Moreover, higher modes can be considered as quasi-statically driven in the considered frequency range, i.e., their electrical equivalent reduces to a capacitance, whose value is [7]:

$C_{n}=\frac{1}{K_{n}}=\frac{L_{s}^{3}}{\lambda_{c c, n}^{4}} \frac{1}{E I_{s}}$

where $E$ is the Young's modulus of the material which the beams are made of and $I_{s}$ is the moment of inertia of the support beam cross-section.

The circuit in Fig. 3 can be further simplified by using the impedance transformation properties of ideal transformers, so that every impedance is transferred to the first loop (Fig. 4). The capacitance $C_{u p}$, taking into account all the higher order modes of both supports, can be expressed as:

$$
C_{u p}=\sum_{n=1}^{\infty} \Pi_{n}^{2} \frac{C_{n}}{2}=\frac{L_{b}^{2} L_{s}}{2 \lambda_{f f, 1}^{2} E I_{s}} \sum_{n=1}^{\infty} \frac{\left(F\left(\lambda_{c c, n}, \lambda_{f f, 1}\right)\right)^{2}}{\lambda_{c c, n}^{2}}
$$

Because of the boundedness of $F$, the terms of the series in Eq. (10) vanish as $\lambda_{c c, n}^{-2}$, so that only a limited number of higher modes of the support beam can be actually used to compute $C_{u p}$.

A further simplification is possible by removing $C_{u p}$ altogether, leading to an equivalent circuit reduced to a single 
Fig. 3 Full equivalent circuit for the resonator

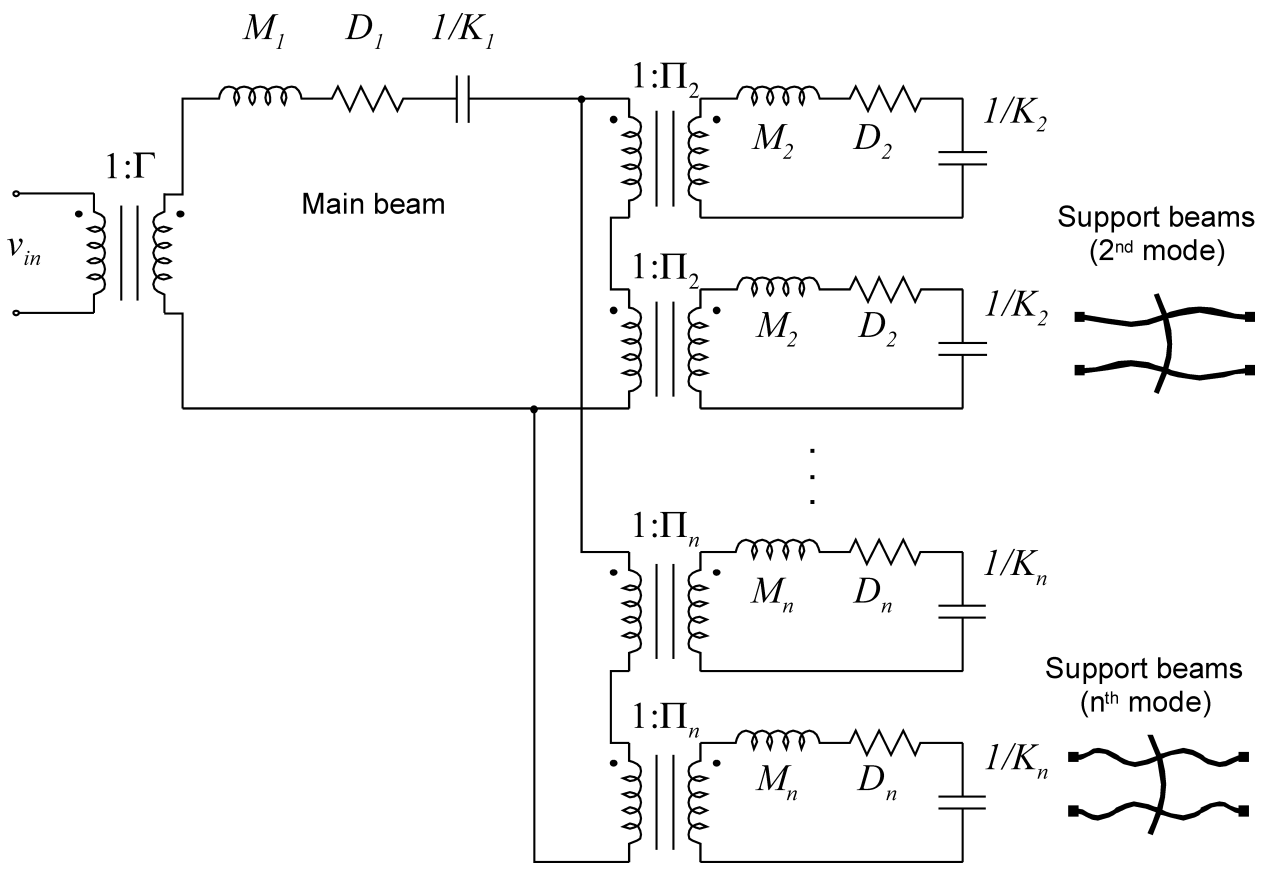

Fig. 4 Reduced equivalent circuit

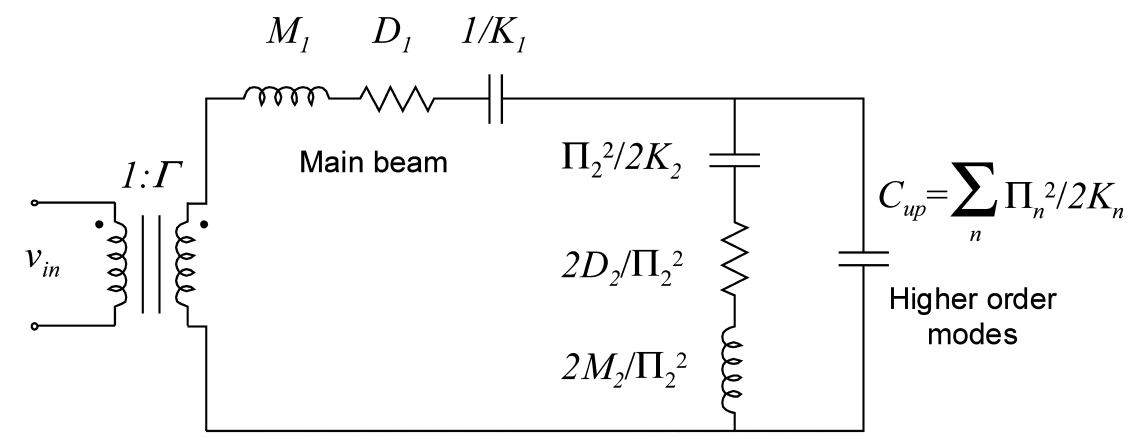

RLC series. In the next section, a comparison between FEM simulations and the results predicted by the circuits with $C_{u p}$ (extended) and without (simplified) will be presented.

\section{FEM simulations}

To validate the model, modal and frequency response simulations were performed using FEMLAB [13], and the results compared with the equivalent circuit predictions. Two types of simulations were implemented: the first one based on 1D beam elements (described by the Euler-Bernoulli theory), the second one with $2 \mathrm{D}$ elements. The simulated device was characterized by the following dimensions (as defined in Fig. (1): $L_{b}=42.2 \mu \mathrm{m}, L_{s}=59.8 \mu \mathrm{m} \div 79.8$ $\mu \mathrm{m}, W_{s}=W_{b}=2.2 \mu \mathrm{m}$. The value $L_{s}=69.8 \mu \mathrm{m}$ corresponds to the matched condition, where the main and support beams show resonance at the same frequency.

For each $L_{s}$, a modal simulation was carried out to extract the resonance frequency of the first mode. Subsequently, a frequency response simulation was performed in a nar- row band around the resonance frequency, and the maximum deflection $w_{\max }$ of the main beam was extracted for each frequency. A uniform distributed mechanical load $p=\Gamma_{1} V_{\text {in }} / L_{e}$ was used to mimic the electromechanical load applied by the electrode, $L_{e}$ being the length of the electrode (see Fig. 1). In the frequency response simulations, the damping was modelled as a bulk viscoelastic loss according to the Kelvin-Voigt model [14]. The same damping model was used to extract the equivalent resistances in the circuit.

Assuming a lumped-parameter second-order resonant system behaviour around the resonance frequency, the simulated frequency response was fitted against this function:

$$
\left|W_{\max }(f)\right|=\left|\frac{\frac{W_{R}}{Q}}{1-\left(\frac{f}{f_{0}}\right)^{2}+j \frac{f}{Q f_{0}}}\right|
$$

where $W_{\max }$ is the Fourier transform of the deflection, $f_{0}$ the resonance frequency, $W_{R}$ the deflection at resonance, and $Q$ the quality factor. $W_{R}$ and $Q$ were used as fitting parameters. 
Simulations results for $f_{0}, Q$ and $W_{R}$ are compared with the corresponding values predicted by the extended and the simplified circuit (as defined at the end of the preceding section) in the graphs of Figs. 5-7. On the horizontal axis, the mismatch, i.e. the difference between the actual and the matched support length, is represented.

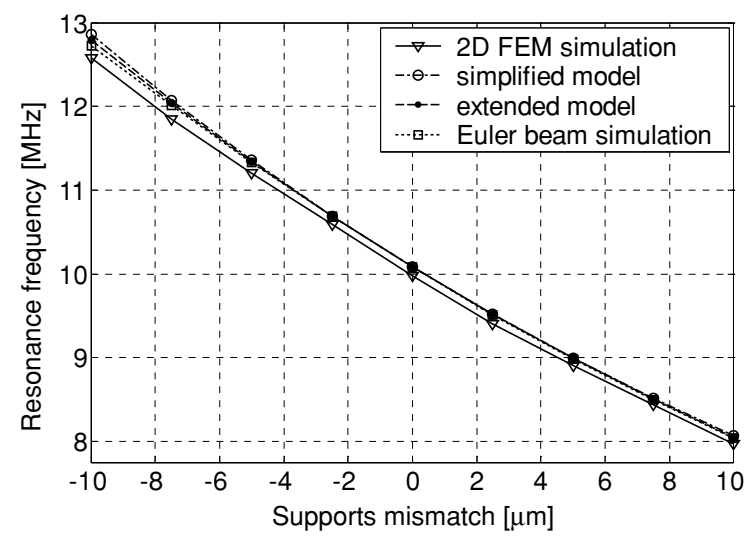

Fig. 5 Frequency resonance $f_{0}$ as a function of support length mismatch: comparison between the equivalent circuit and simulation results

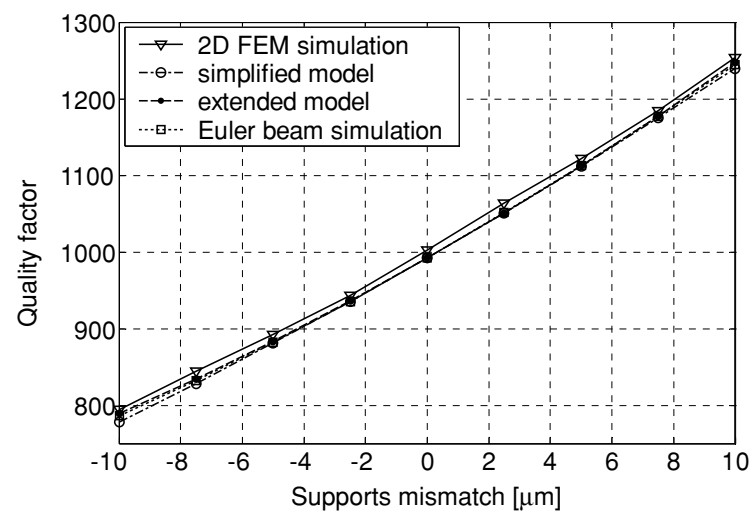

Fig. 6 Quality factor $Q$ as a function of support length mismatch: comparison between the equivalent circuit and simulation results

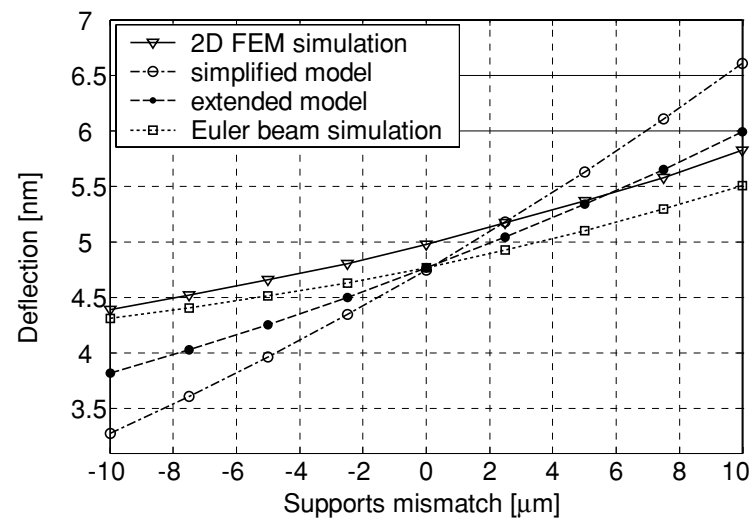

Fig. 7 Maximum displacement $W_{R}$ of the main beam as a function of support length mismatch: comparison between the equivalent circuit and simulation results
The resonance frequency and quality factor are more accurately predicted by the extended circuit model than by the simplified one (Figs. 5 and 6), but the relative error is very small in both cases (less than $2.5 \%$ with respect to $2 \mathrm{D}$ simulations for a large relative mismatch of $\pm 10 \mu \mathrm{m}$ over $69.8 \mu \mathrm{m})$.

For the maximum deflection $W_{R}$ (Fig. 7) the error between theoretical and simulated curves is significantly larger, especially if we consider the simplified model. The maximum relative error with respect to the $2 \mathrm{D}$ simulations is nearly $13 \%$ for the extended model, and nearly $25 \%$ for the simplified one. The total error is contributed both by an offset and a higher slope of the curve.

The offset is to be ascribed to the use of the Euler-Bernoulli equation in deriving the equivalent circuit, as the 1D simulations (which are based on the same model) are also affected by.

\section{Conclusions}

An equivalent electrical circuit describing the behaviour of a free-free flexural MEMS resonator was developed, and its predictions compared with the results of FEM simulations. The circuit, even in a simplified topology, correctly predicts the values of the resonance frequency and the quality factor of the device, even when possible fabrication defects alter the matching condition used in the design. Acceptable results are obtained with respect to the prediction of the maximum resonator deflection (which is related to the output signal amplitude, and the linearity of the device) only when the extended equivalent circuit is used. This effect can be ascribed, at least to some extent, to the non-ideal behaviour of the real device with respect to the Euler-Bernoulli model.

\section{References}

1. Fedder, G.K., Jing, Q.: IEEE Trans. Circuits and Systems-II, 46 1309-1315 (1999)

2. Courtois, B., Karam, J.M., Mir, S., Lubaszewski, M., Szekely, V., Rencz, M., Hofmann, K., Glesner, M.: Proceedings, Twelfth International Conference on VLSI Design, Jan. 10, 270-275 (1999)

3. Fedder, G.K.: Proceedings, International Workshop on Behavioral Modeling and Simulation, (BMAS 2003), Oct. 7(8), (2003) 6469

4. Nguyen, C.T.-C.: Dig. Of Papers, Topical Meeting on Silicon Monolithic Integrated Circuits in RF Systems, Sep. 12-14. (2001), pp. 23-32

5. Mattila, T., Kiihamäki, J., Lamminmäki, T., Jaakkola, O., Rantakari, P., Oja, A., Seppä, H., Kattelus, H., Tittonen, I.: Sens. Actuators A, 101, 1-9 (2002) 
6. Lee, S., Demirci, M.U., Nguyen, C.T.-C.: Digest of Technical Papers, the $11^{\text {th }}$ Int. Conf. on Solid-State Sensors \& Actuators (Transducers'01), Jun. 10-14. (2001) pp. 1094-1097

7. Tilmans, H.A.C.: J. Micromech. Microeng. 7, 285-309 (1997)

8. Bannon, F.D., Clark, J.R., Nguyen, C.T.-C.: J. Solid State Circuits 35, 512-526 (2000)

9. Paci, D., Nannini, A., Pieri, F.: Symposium on Design, Test, Integration of MEMS/MOEMS (DTIP2005), 1-3 June (2005), pp. 195-199
10. Hsu, W.-T., Clark, J.R., Nguyen, C.T.-C.: Digest of Technical Papers, the 11th Int. Conf. on Solid-State Sensors \& Actuators (Transducers'01), Jun. 10-14. (2001), pp. 1110-1113

11. Marguerre K., Wolfel, K.: Mechanics of Vibrations. Sijthoff \& Noordhoff (1979)

12. Wang, K., Wong, A.-C., Nguyen, C.T.-C,: IEEE J. Microelectromech. Syst. 9(3), 347-360 (2000)

13. http://www.comsol.com/products/femlab/

14. Fung, Y.C.: Foundations of Solid Mechanics, Prentice Hall (1965) 